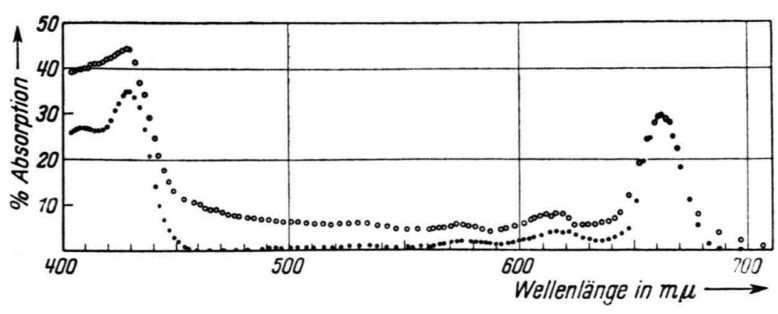

Abb. 4. Prozentuale Absorption des gelbgrünen Pigments von Vaucheria synandra (offene Kreise), zum Vergleich diejenige von reinem Chlorophyll a, umgerechnet auf gleichstarke Absorption im Rotmaximum (gefüllte Kreise).

sorption im Rot in den übrigen Spektralbereichen stärker, vor allem im Blau und im Blaugrün. Die ätherische Lösung dieses gelbgrünen Farbstoffes zeigte mit konzentrierter Schwefelsäure und mit Salzsäure keine Carotinoid-Reaktion (KARRER und JUK-

10 P. Karrer u. E. Jucker, Carotinoide, Verlag Birkhäuser, Basel 1948.
${ }_{\text {KER }}{ }^{10}$ ). Der Phasentest (PAECH und TraceY ${ }^{11}$ ), Überschichtung von $\mathrm{KOH}$-haltigem Methanol mit der ätherischen Farbstofflösung und anschließender Durchmischung der beiden Lösungen, ergab eine vollkommen farblose Ätherlösung über dem zunächst gelb, dann schwach grüngelb gefärbten Methylalkohol, also auch kein Anzeichen für Vermischung mit Carotinoiden. - Da die Absorptionsmaxima an denselben Stellen liegen, wie diejenigen von reinem Chlorophyll a, handelt es sich hier wohl auch nicht um Allomerisationsprodukte von Chlorophyll a. Es ist vermutlich Chlorophyll a, welches an andere Stoffe gebunden ist und wurde wohl oft wegen seiner dem Chlorophyll b ähnlichen Färbung für Chlorophyll b gehalten.

Fräulein B. Meister und I. Würzberg danke ich für die Mithilfe bei den Versuchen.

11 K. Paech u. M. V. Tracey, Moderne Methoden der Pflanzenphysiologie, Springer, Berlin-Göttingen-Heidelberg 1955.

\title{
Über das Vorkommen von Djenkolsäure und einer C-S-Lyase in den Samen von Albizzia lophantha Benth. (Mimosaceae)
}

\author{
Von Rolf Gmelin, Gerhard Hasenmaier und Günther Strauss \\ Aus dem wissenschaftlichen Laboratorium der Robugen G. m. b. H., Eßlingen am Neckar \\ (Z. Naturforschg. 12 b, 687-697 [1957]; eingegangen am 23. Juli 1957)
}

Es wird das Vorkommen eines neuen Enzyms, einer C-S-Lyase, und seines natürlichen Substrates, der Djenkolsäure, in den Samen von Albizzia lophantha Benth. (Mimosaceae) beschrieben.

Djenkolsäure wird durch das Enzym in Brenztraubensäure, Ammoniak und einen lauchartig riechenden Stoff, bei dem es sich wahrscheinlich primär um das sehr instabile Methylendithiol handelt, gespalten.

Das Albizzia-Enzym spaltet außerdem S-Alkyl-L-cysteine und Cystathionin, $S$-Alkyl-L-cysteinsulfoxyde und L-Cystein.

Bei der Spaltung der S-Alkyl-L-cysteine entstehen Brenztraubensäure, Ammoniak und Mercaptane, Cystathionin zerfällt in Brenztraubensäure, Ammoniak und Homocystein, die $S$-Alkyl-L-cysteinsulfoxyde werden in Brenztraubensäure, Ammoniak und Alkylthiosulfinsäurealkylester gespalten.

Die Spaltung von L-Cystein, bei der Ammoniak und $\mathrm{H}_{2} \mathrm{~S}$ gebildet werden, ist im einzelnen noch ungeklärt.

Es werden einige Alkyl-thiamindisulfide beschrieben, die durch Umsetzung der enzymatisch gebildeten Alkylthiosulfinsäurealkylester mit Thiamin bei $p_{\mathrm{H}} 8$ gewonnen wurden. Aus $S$-Benzyl-Lcysteinsulfoxyd ließ sich durch enzymatischen Abbau Benzylthiosulfinsäurebenzylester kristallin erhalten.

Durch enzymatische Spaltung von $S$-Methyl-pL-cystein wurde $S$-Methyl-D-cystein gewonnen.

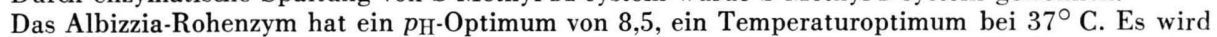
durch Pyridoxalphosphat aktiviert und durch Carbonylreagentien inaktiviert. Durch Glycin wird es sehr stark gehemmt.

Alle bisher im Pflanzenreich bekannten S-haltigen Aminosäuren leiten sich von L-Cystein und L-Methionin ab. Ihre Zahl ist - einschließlich der noch nicht restlos aufgeklärten Vertreter - heute auf etwa 15 angestiegen.

1 a A. G. van Veen u. A. J. Hyman, Geneeskund Tijdschr. Nederl. Indië 73, 991 [1933].
Zur Orientierung sei einleitend eine kurze Übersicht über diese Cystein- und Methioninderivate gebracht.

Aus der Djenkolbohne (Pithecolobium lobatum und P. bigeminum [Mimosaceae]) isolierten van VeEn und Hyman $^{1}$ eine Aminosäure, die sie Djenkolsäure nannten. Die von den Autoren für Djenkolsäure vorgeschla-

1 b A. G. van Veen u. A. J. Hyman, Rec. Trav. bot. néerl. 54, 493 [1935]. 
gene Formulierung als L-Cysteinthioacetal des Formaldehyds konnten DU Vigneaud u. a. bestätigen ${ }^{2}$ und durch Synthese beweisen ${ }^{3}$. Nach eigenen Untersuchungen ${ }^{4}$ kommt Djenkolsäure auch in Pithecolobium multiflorum und in P. dulce vor. Es ist anzunehmen, daß sie eine in Mimosaceen-Arten weitverbreitete Aminosäure ist.

Aus den Zwiebeln von Allium sativum und Allium ursinum (Liliaceae) gewannen Stoll und Seebeck ${ }^{5}$ Alliin, (+)S-Allyl-L-cysteinsulfoxyd, und klärten die enzymatische Bildung des antibiotisch wirksamen Allicins aus dieser Vorstufe auf. Matsukawa u. a. ${ }^{6}$ schlossen aus dem Nachweis von Methylthiosulfinsäuremethyl ester und Propylthiosulfinsäurepropylester, daß auch $S$-Methyl- und $S$-Propyl-L-cysteinsulfoxyd in diesen und einigen anderen Allium-Arten enthalten sein müssen. Einen indirekten Beweis für die Existenz einer ähnlich gebauten Aminosäure, die ebenfalls enzymatisch gespalten wird, in der Küchenzwiebel (Allium cepa, Liliaceae) fand $\mathrm{V}_{\text {ILKKI }}{ }^{7}$ bei papierchromatographischen Studien. Virtanen ${ }^{8}$ gewann aus dem Säurehydrolysat der Küchenzwiebel eine S-haltige Aminosäure der Bruttoformel $\mathrm{C}_{6} \mathrm{H}_{11} \mathrm{O}_{2} \mathrm{NS}$, der er die vorläufige Formulierung $\mathrm{C}_{3} \mathrm{H}_{5}-\mathrm{S}-\mathrm{CH}_{2}-\mathrm{CH}\left(\mathrm{NH}_{2}\right)-\mathrm{COOH}$ gab.

2 weitere $\mathrm{S}$-haltige Aminosäuren wurden in BrassicaArten entdeckt:

$S$-Methyl-sulfonium-methionin (McRorie u. a. ${ }^{9}$ ) und $(+) S$-Methyl-L-cysteinsulfoxyd (Synge und Wood ${ }^{10}$ und unabhängig davon Morris und Thompson $\left.{ }^{11}\right)$. Letzteres soll nach VIRTANen ${ }^{12}$ auch in anderen CruciferenArten vorkommen. Aus den Samen der Gartenbohne (Phaseolus vulgaris, Papilionaceae) isolierten Тномеson und Zacharius ${ }^{13}$ (-) $S$-Methyl-L-cystein.

Die gleiche Aminosäure wurde auch als natürlicher Anabolit in Neurospora crassa angetroffen (RAYland und Liverman ${ }^{14}$ ), für die sie Wuchsstoffeigenschaften $\mathrm{zu}$ besitzen scheint.

Cantoni $^{15}$ erkannte im „aktiven Methionin“, $S$-Adenosyl-L-methionin, die für einige TransmethylierungsReaktionen verantwortliche energiereiche Form des

2 V. du Vigneaud u. W. J. Patterson, J. biol. Chemistry 114, 535 [1936].

3 M. D. Armstrong u. V. du Vigneaud, J. biol. Chemistry 168, 373 [1947].

4 R. Gmelin u. G. Hasenmaier, unveröffentlicht.

5 a A. Stoll u. E. Seевeck, Helv. chim. Acta 31, 189 [1948].

5 b A. Stoll u. E. Seebeck, Helv. chim. Acta 32, 197 [1949].

5 c A. Stoll u. E. Seевеск, Scientia pharmac. 18, 61 [1950].

5 d A. Stoll u. E. Sеeвеск, Experientia [Basel] 6, 330 [1950].

6 T. Matsukawa, S. Yurugi u. T. Matsuoka, Science [Washington] 118, 109 [1953].

7 P. VILKki, Suomen Kemistilethi B 27, 21 [1954].

8 A. I. Virtanen u. E. Mattikkala, Suomen Kemistilehti B 29, 134 [1956].

9 R. A. McRorie, G. L. Sutherland, M. S. Lewis, A. D. Barton, M. R. Glazener u. W. Shive, J. Amer. chem. Soc. 76, 115 [1954].

10 R. L. M. Synge u. J. C. Wood, Biochem. J. 60, 15 [1955].

11 C. J. Morris u. J. F. Thompson, J. Amer. chem. Soc. 78, $1605[1956]$.
L-Methionins. Vermutlich spielt diese Verbindung auch in der Pflanze, in der Methylierungs-Prozesse besonders häufig sein müssen - man denke an die vielen Naturstoffe mit $\mathrm{CH}_{3}-\mathrm{O}-, \mathrm{CH}_{3}-\mathrm{N}-$ und $\mathrm{CH}_{3}-\mathrm{S}$-Gruppen -, eine ähnliche Rolle wie im tierischen Organismus.

Cystathionin nimmt eine Zwischenstellung zwischen Methionin, Homocystein und Cystein ein ${ }^{16}$. Es ist bei der Umbildung dieser Stoffe nach folgendem Reaktionsschema beteiligt:

1. Methionin $-\mathrm{CH}_{3} \rightarrow$ Homocystein,

2. Homocystein + Serin $\rightarrow$ Cystathionin,

3. Cystathionin $\rightarrow$ Cystein + Homoserin.

Der Vollständigkeit halber gehören in diese Aufzählung 2 weitere Di-aminosäuren mit einer Thioätherbrücke: das als solches noch nicht isolierte $S$ - $\alpha$ ( $\beta$-Indolylalanin) L-cystein, ein Baustein des Knollenblätterpilz-Giftstoffes Phalloidin, und der noch hypothetische, ähnlich gebaute Baustein der Amanitine, mit deren Aufklärung sich zur Zeit Wieland und Mitarbb. ${ }^{17}$ befassen.

Auf Grund der wichtigen biochemischen Funktionen der S-haltigen Aminosäuren sind natürlich auch die an ihrem Auf- und Abbau beteiligten Enzymreaktionen von größtem Interesse - vor allem solche, bei denen $\mathrm{C}$ - S-Bindungen gebildet oder gelöst werden.

Eine Cysteinase aus Proteus vulgaris wurde erstmals durch $\mathrm{T}_{\mathrm{ARR}}{ }^{18}$ beschrieben. Ähnliche Enzyme wurden später auch in anderen Mikroorganismen angetroffen: in E.coli (Fromageot und DesNUELLE ${ }^{19}$ ), in Bacillus subtilis (Fromageot und $\mathrm{KIUN}^{20}$ ) und in Propionibacterium pentosaceum (Desnuelle u. a. ${ }^{21}$ ). Fromageot ${ }^{22}$ und Smythe ${ }^{23}$ berichteten über Desulfhydrasen tierischen Ursprungs. Binkley ${ }^{24}$ beschrieb eine Desulfhydrase mit einem $p_{\mathrm{H}}$-Optimum von $6,8-7,2$, die Cystein

12 A. I. Virtanen, Festschrift Arthur Stoll, 575, Birkhäuser, Basel 1957.

13 J. F. Thompson u. R. M. Zacharius, Nature [London] 178, 593 [1956].

14 J. B. Rayland u. J. L. Liverman, Arch. Biochem. Biophysics 65, 574 [1956].

15 G. L. Cantoni, J. Amer. chem. Soc. 74, 2942 [1952]; J. biol. Chemistry 189, 745 [1951].

16 E. S. West u. W. R. Todd, Textbook of Biochemistry, 1126 , Macmillan Co., New York 1955.

17 Th. Wieland, Angew. Chem. 69, 44 [1957].

18 H. L. A. TARR, Biochem. J. 27, 1869 [1933].

19 C. Fromageot u. P. Desnuelle, Enzymologia [Den Haag] 6, 80 [1939].

20 C. Fromageot u. T. P. Kiun, Bull. Soc. Chim. Biol. 23, 1471 [1941].

21 P. Desnuelle, E. Wookey u. C. Fromageot, Enzymologia [Den Haag] 8, 225 [1940].

22 C. Fromageot, E. Wookey u. P. Chaix, Enzymologia [Den Haag] 9, 198 [1940].

23 C. V. Smythe, J. biol. Chemistry 142, 387 [1942].

24 F. Binkley, J. biol. Chemistry 150, 261 [1943]. 
spaltet, außerdem ein Enzym aus Rattenleber, das Cystein und gleichzeitig einige Thioäther-Derivate spaltet $^{25}$. An einer Enzymzubereitung aus Proteus morgani, die aus Cystein äquimolare Mengen von Brenztraubensäure, Ammoniak und $\mathrm{H}_{2} \mathrm{~S}$ bildet, stellte Kaldio ${ }^{26}$ eine Aktivierung durch Pyridoxalphosphat fest. Eine entsprechende Aktivierung erfährt ein methionin-spaltendes Enzym aus Clostridium sporogenes (Wiesendanger und Nisman ${ }^{27}$ ).

Einen neuen Typ enzymatischer $\mathrm{C}$-S-Spaltung beschrieb vor kurzem Cantoni ${ }^{28}$ : ein Enzym der Alge Polysiphonia lanosa bildet aus dem in der gleichen Art vorkommenden Propiothetin Dimethylsulfid und Acrylsäure.

In höheren Pflanzen war die von Stoll und SEEBECK $^{29}$ aufgefundene und eingehend untersuchte Alliinase das einzige in diese Gruppe gehörende Enzym. Ihre Spezifität beschränkt sich jedoch auf Aminosäuren vom Typ (+)S-Alkyl-L-cysteinsulfoxyd. Wie Gorjatschenkowa ${ }^{30}$ feststellte, ist Pyridoxalphosphat das Coferment der Alliinase.

Vor kurzem gelang MATsuo $^{31}$ die Isolierung eines kristallinen Enzyms aus Rattenleber, das Cystathionin in Cystein, Ammoniak und a-Ketobuttersäure zerlegt.

Es seien in diesem Zusammenhang noch die erst kürzlich beschriebenen enzymatischen Synthesen von S-Methyl-L-cystein aus L-Serin und Methanthiol (Wolf und B BaCK ${ }^{32}$ ) und von L-Cystein aus L-Serin und $\mathrm{H}_{2} \mathrm{~S}$ (Schlossmann und Lynen ${ }^{33}$ ) erwähnt.

In dieser Arbeit beschreiben wir die Auffindung eines neuen Enzyms aus dieser Gruppe sowie die Isolierung seines natürlichen Substrats Djenkolsäure aus den Samen von Albizzia lophantha Benth. (Mimosaceae).

Über Inhaltstoffe und Enzyme in Albizzia-Arten sind in der Literatur nur vereinzelte Angaben zu finden. Nach Virtanes ${ }^{34}$ ist in den Samen von $\mathrm{Al}$ bizzia lophantha Benth. 4-Hydroxypipecolinsäure enthalten.

Für Albizzia lebbek Benth. sind Monobutyrase und Urease als Enzyme angegeben $\left(\mathrm{T}_{\mathrm{ANAKA}}{ }^{35}\right)$. Als

${ }^{25}$ F. Binkley, J. biol. Chemistry 186, 287 [1950].

26 R. E. Kallio, J. biol. Chemistry 192, 371 [1951].

27 S. Wiesendanger u. B. Nisman, C. R. hebd. Séances Acad. Sci. 237, 764 [1953].

28 G. L. Cantoni u. D. G. Anderson, J. biol. Chemistry 222, 171 [1956].

29 A. Stoll u. E. Seebeck, Advances in Enzymol. 11, 377 [1951].

30 E. V. Gorjatschenkowa, Doklady Akadem. Nauk SSSR 87, 457 [1952]. einen Hinweis auf S-haltige Verbindungen in diesen und verwandten Arten kann man den knoblauchartigen Geruch auffassen, der in vielen MimosaceenArten auftritt ${ }^{36}$ : Pithecolobium bigeminum, Albizzia luzida, Acacia farnesiana, Parkia speciosa und andere. Über die chemische Natur der verantwortlichen Verbindungen und über die mögliche Beteiligung von Enzymen bei der Geruchsbildung scheinen jedoch bisher keine Angaben vorzuliegen.

Beim Behandeln des Samenpulvers von Albizzia lophantha mit kaltem Wasser trat ein starker und unangenehmer Geruch auf, der deutlich an den Geruch zerriebener Küchen- oder Knoblauchzwiebeln erinnerte. Mit Methanol, Äthanol oder verdünnter Salzsäure blieb die Bildung des Geruchsstoffes aus. Nahm man jedoch den Rückstand eingedampfter methanolischer Auszüge aus den Samen in Wasser auf und versetzte die Lösung mit einer Proteinfällung, die man durch Zugabe von überschüssigem Alkohol zu einem wäßrigen Auszug der Samen erhalten konnte, so trat nach kurzer Zeit wieder der charakteristische Geruch auf.

Eine papierchromatographische AminosäurenAnalyse des methanolischen Auszugs der Samen ließ eine Anzahl von Aminosäuren erkennen - darunter eine Aminosäure, die auf dem Papierstreifen nach einigen Stdn. Jodazidlösung entfärbte. Sie zeigte in diesem Verhalten Ähnlichkeit mit Methionin und $S$-Methylcystein, wies jedoch einen wesentlich geringeren $R_{f}$-Wert auf. Mit den wäßrigen Auszügen der Albizzia-Samen ließ sich papierchromatographisch gut verfolgen, wie diese Aminosäure im Verlauf von einigen Stdn. restlos verschwand.

Folgende Beobachtungen ergaben weitere Anhaltspunkte für einen enzymatischen Prozeß:

1. Jodazidlösung, die vom methanolischen Auszug der Albizzia-Samen nur sehr träge entfärbt wird, wurde vom wäßrigen Auszug spontan entfärbt. Die Geschwindigkeit der Entfärbung nahm mit der Zeit $\mathrm{zu}$.

2. Der wäßrige Auszug gab mit zunehmender Zeit eine immer intensiver werdende Rotfärbung mit

31 Y. Matsuo, Feder. Proc. 16, 218 [1957].

32 E. C. Wolf u. S. Black, J. Amer. chem. Soc. 78, 5868 [1956].

33 K. Schlossmann u. F. Lynen, Angew. Chem. 69, 179 [1957].

34 A. I. Virtanen, loc. cit. ${ }^{12}$.

35 S. TaNAKA, J. agric. chem. Soc. Japan, Bull. 6, 454 [1930], zit. nach C. A. 1930, 4075.

36 F. Czapek, Biochemie der Pflanzen, 2. Aufl., Bd. 3, G. Fischer, Jena 1921; O. W Bibliographisches Institut, Leipzig 1923. 
alkalischer Nitroprussid-Natrium-Lösung. Der methanolische Auszug gab keine Farbreaktion mit diesem Reagenz.

3. Im wäßrigen Auszug ließen sich nach einiger Zeit Brenztraubensäure und Ammoniak nachweisen. Im methanolischen Auszug treten diese Stoffe erst nach Zugabe der gelösten Proteinfällung zum Trokkenrückstand auf.

Die Darstellung der fraglichen Aminosäure erfolgte zunächst durch Ionenaustauscher. Hierbei konnte eine geringe Menge eines Stoffes gewonnen werden, der in allen Eigenschaften mit Djenkolsäure übereinstimmte und durch die Proteinlösung in Brenztraubensäure, Ammoniak und einen zwiebelartig riechenden Stoff zerlegt wurde.

Um eine größere Menge dieser Aminosäure zu gewinnen., wurden die Samen von Albizzia lophantha nach der Methode von van VEen und Hyman ${ }^{37}$ aufgearbeitet. Wir erhielten dabei 1,6g Djenkolsäure. Da sich Djenkolsäure nur sehr schwer und nur unter Zersetzung umkristallisieren ließ, was auch van VeEN und Hyman beobachtet hatten, erhielten wir keine genauen Analysenwerte bei der Verbrennung. Unter Berücksichtigung eines unverbrennbaren Rückstands errechneten wir die Werte: C $32,43 \%$, H 8,76\%, $\mathrm{N} 11,61 \%$, S 23,04\% (ber. für $\mathrm{C}_{7} \mathrm{H}_{14} \mathrm{~N}_{2} \mathrm{~S}_{2} \mathrm{O}_{4}$ C $33,06 \%, \quad H 5,54 \%, \quad \mathrm{~N} 11,01 \%, \quad \mathrm{~S} 25,21 \%)$. Die Identität mit Djenkolsäure ergab sich zusätzlich durch papierchromatographischen Vergleich in verschiedenen Lösungsmitteln: der aus Albizzia lophantha isolierte Stoff zeigte in allen Lösungsmitteln gleiche $R_{f}$-Werte wie synthetische Djenkolsäure. Auch in ihrem Verhalten gegen das Albizzia-Enzym stimmten die beiden Stoffe völlig überein.

Aus der Bestimmung von enzymatisch gebildetem Ammoniak ließ sich für das von den Samenschalen befreite Samenpulver von Albizzia lophantha ein Djenkolsäuregehalt von etwa $2,1 \%$ ermitteln, für die ganzen Samen etwa 1,1 Prozent.

Es war uns bisher noch nicht möglich, den bei der enzymatischen Spaltung von Djenkolsäure neben Brenztraubensäure und Ammoniak gebildeten Thiolkörper, der für den unangenehmen Geruch verantwortlich ist, zu erfassen.

Er scheint sehr labil zu sein, denn er läßt sich durch Wasserdampf nicht unzersetzt übertreiben und zerfällt schon bei Raumtemperatur nach einiger Zeit unter $\mathrm{H}_{2} \mathrm{~S}$-Abspaltung.

37 A. G. van Veen u. A. J. Hyman, loc. cit. ${ }^{1}$.
In Analogie zu ähnlichen enzymatischen Spaltungen von Cysteinderivaten unter Ammoniak-, Brenztraubensäure- und Thiolbildung nimmt die Djenkolsäure-Spaltung vermutlich folgenden Verlauf:

$$
\begin{aligned}
\mathrm{H}_{2} \mathrm{C}{ }_{\mathrm{S}-\mathrm{CH}_{2}-\mathrm{CH}\left(\mathrm{NH}_{2}\right) \mathrm{COOH}}^{\mathrm{S}-\mathrm{CH}_{2}-\mathrm{CH}\left(\mathrm{NH}_{2}\right) \mathrm{COOH}} \quad \text { (Albizzia-Enzym) } \\
\mathrm{H}_{2} \mathrm{C}{ }_{\mathrm{SH}}^{\mathrm{SH}}+2 \mathrm{CH}_{3} \mathrm{COCOOH}+{ }_{2} \mathrm{NH}_{3} .
\end{aligned}
$$

Das nach dieser Formulierung gebildete Methylendithiol zerfällt in $\mathrm{H}_{2} \mathrm{~S}$ und einen wiederum unbeständigen Körper (Thioformaldehyd [?]):

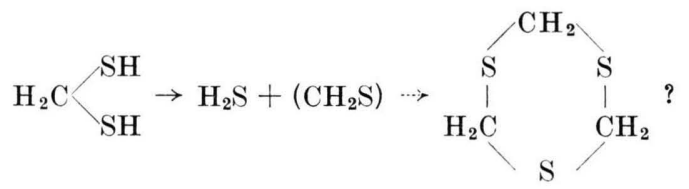

Abb. 1 zeigt schematisch die papierchromatographische Aminosäuren-Analyse der Albizzia lophantha-Samen, das Verschwinden von Djenkolsäure durch einen enzymatischen Prozeß und die gleichzeitige Neubildung von Brenztraubensäure.

Nachdem auf diese Weise gezeigt werden konnte, daß in den Samen von Albizzia lophantha Djenkol-

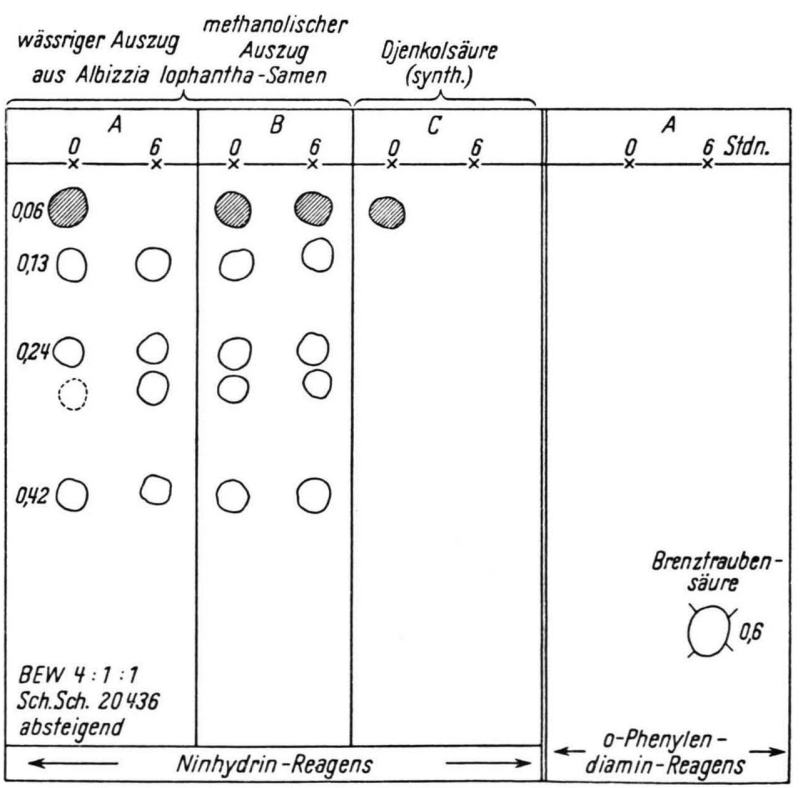

Abb. 1. Papierchromatographischer Nachweis der enzymatischen Spaltung von Djenkolsäure. A: Wäßriger Auszug aus Albizzia lophantha-Samen. B: Methanolischer Auszug aus Albizzia lophantha-Samen. C: Synthetische Djenkolsäure +Albizzia-Enzym. 
säure und gleichzeitig ein djenkolsäurespaltendes Enzym vorkommt, erhob sich die Frage der Spezifität dieses Enzyms. Mit einer Rohenzymzubereitung (s. experimenteller Teil) wurde zunächst eine Anzahl S-substituierter Cysteinderivate durchgeprüft: $S$-Methyl-, $S$-Äthyl-, $S$-Propyl-, $S$-Allyl- und $S$-Benzyl-L-cystein.

Bei allen genannten Cysteinderivaten trat eine Spaltung ein. In der Reaktionsmischung ließen sich Brenztraubensäure, Ammoniak und die jeweiligen, charakteristisch riechenden Merkaptane nachweisen.

Bei $S$-Methyl-DL-cystein konnte im Gegensatz zu $S$-Methyl-L-cystein, bei dem die Spaltung, gemessen an der Ammoniak- und Brenztraubensäure-Bildung etwa $90 \%$ betrug, nur eine 45 -proz. Spaltung festgestellt werden. Dies deutet darauf hin, daß nur die L-Formen der obengenannten Cysteinderivate gespalten werden. Eine Bestätigung dafür war die be. queme Isolierung von $S$-Methyl-D-cystein aus der Reaktionsmischung. Für die enzymatische Spaltung von $S$-Alkyl-L-cystein-Derivaten gilt vermutlich das Reaktionsschema:

$$
\begin{aligned}
& \mathrm{R}-\mathrm{S}-\mathrm{CH}_{2}-\mathrm{CH}\left(\mathrm{NH}_{2}\right) \mathrm{COOH} \begin{array}{l}
\text { Albizzia-Enzym } \\
\mathrm{H}_{2} \mathrm{O}
\end{array} \\
& \mathrm{R}-\mathrm{SH}+\mathrm{CH}_{3} \mathrm{COCOOH}+\mathrm{NH}_{3} .
\end{aligned}
$$

Erwartungsgemäß wurde Cystathionin in gleicher Weise in Brenztraubensäure, Ammoniak und Homocystein gespalten

$$
\begin{aligned}
& \text { S } \begin{array}{lc}
\mathrm{CH}_{2}-\mathrm{CH}\left(\mathrm{NH}_{2}\right) \mathrm{COOH} & \text { Albizzia-Enzym } \\
\mathrm{CH}_{2}-\mathrm{CH}_{2}-\mathrm{CH}\left(\mathrm{NH}_{2}\right) \mathrm{COOH} & \mathrm{H}_{2} \mathrm{O}
\end{array} \\
& \begin{array}{c}
\mathrm{CH}_{3} \mathrm{COCOOH}+\mathrm{NH}_{3} \\
+\underset{\mathrm{HS}}{+}-\mathrm{CH}_{2}-\mathrm{CH}_{2}-\mathrm{CH}\left(\mathrm{NH}_{2}\right) \mathrm{COOH} .
\end{array}
\end{aligned}
$$

Da es sich um eine Mischung von DL- und alloCystathionin gehandelt hatte, trat nur eine partielle Spaltung ein (Resistenz der D-Form gegen das Enzym).

In Abb. 2 wird papierchromatographisch die Spaltung von Cystathionin und die enzymatische Bil. dung von Homocystein und Brenztraubensäure demonstriert.

Wir haben also in dieser Spaltung ein Gegenstück zur „normalen“ Cystathionin-Spaltung durch ein Enzym der Leber unter Bildung von Cystein, Ammoniak und $\alpha$-Ketobuttersäure.

Lanthionin scheint ebenfalls durch das AlbizziaEnzym gespalten zu werden, denn es ließ sich Ammoniak nachweisen $(25 \%$ der Theorie) und im PC trat ein neuer Fleck auf, der mit Ninhydrin positiv reagierte $\left[R_{f}\right.$-Wert in Butanol-Essigsäure-Wasser $4: 1: 1 \quad 0,25$ (aufsteigend) ]. Brenztraubensäure war jedoch nicht nachzuweisen. Der Verlauf dieser Spaltung ist noch ungeklärt.

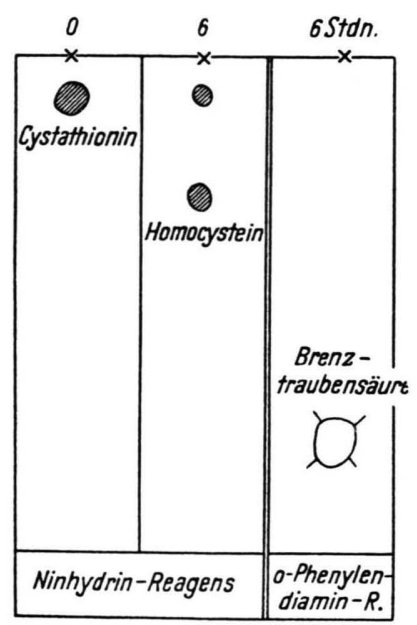

Abb. 2. DL- und allo-Cystathionin + Albizzia-Enzym.

Abb. 3 zeigt den zeitlichen Verlauf der Spaltung von $S$-Alkyl-L-cysteinen durch das Albizzia-Enzym bei $p_{\mathrm{H}} 8,5$. Die prozentuale Spaltung wurde durch gravimetrische Brenztraubensäure-Bestimmung und durch Titration des gebildeten Ammoniaks ermittelt.

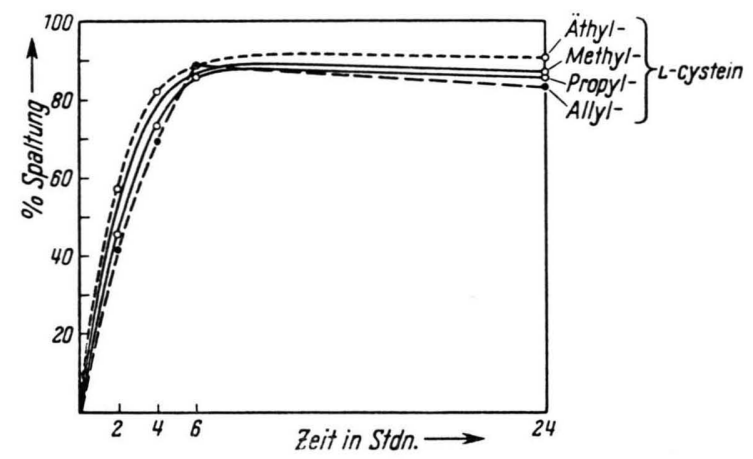

Abb. 3. Zeitlicher Verlauf der enzymatischen Spaltung von $S$-Alkyl-L-cysteinen. $n / 20$-Lösungen in Boratpuffer $p_{\mathrm{H}} 8,5$; 0,5\% Rohenzym; Raumtemperatur.

$S$-Benzyl-L-cystein und Djenkolsäure wurden wegen ihrer geringen Löslichkeit in Wasser nur qualitativ untersucht.

Ein weiteres Substrat für das Albizzia-Enzym ist L-Cystein. Auch hier ist der Verlauf der Spaltung noch nicht geklärt. Als Spaltprodukte ließen sich 
Ammoniak und Schwefelwasserstoff, aber keine Brenztraubensäure nachweisen. Die Cysteinspaltung soll Gegenstand späterer Untersuchungen sein.

Die Vielseitigkeit des Albizzia-Enzyms zeigte sich bei der Prüfung von $S$-Methyl-, $S$-Äthyl-, $S$-Propyl-, $S$-Allyl- und $S$-Benzyl-L-cysteinsulfoxyd, denn auch diese Verbindungen wurden gespalten. Hier verläuft die Reaktion nach dem gleichen Schema, das Stoll und SeEвeck für die Alliinspaltung angegeben hatten:

$$
\begin{aligned}
& \begin{array}{cc}
2 \mathrm{R}-\underset{\downarrow}{\mathrm{S}}-\mathrm{CH}_{2}-\mathrm{CH}\left(\mathrm{NH}_{2}\right) \mathrm{COOH} & \text { Albizzia-Enzym } \\
\stackrel{\mathrm{O}}{\cdots} & \mathrm{H}_{2} \mathrm{O} \\
&
\end{array} \\
& \begin{array}{c}
(2 \mathrm{R}-\mathrm{SH})+2 \mathrm{CH}_{3} \mathrm{COCOOH}+2 \mathrm{NH}_{3} \\
\stackrel{\downarrow}{\mathrm{O}} \\
\stackrel{\mathrm{R}}{\downarrow}-\mathrm{S}-\mathrm{R}+\mathrm{H}_{2} \mathrm{O} \\
\downarrow \\
\mathrm{O}
\end{array}
\end{aligned}
$$

Auch S-Benzyl-L-cysteinsulfoxyd, gegen das Alliinase indifferent ist, wird gespalten: Als Spaltprodukt ließ sich Benzylthiosulfinsäurebenzylester isolieren (Schmp. $81-83^{\circ} \mathrm{C}$ nach Umkristallisieren aus Äther-Petroläther).

Da sich die übrigen Alkylthiosulfinsäurealkylester, die alle einen sehr scharfen Geschmack besitzen, wegen ihrer leichten Zersetzlichkeit schlecht durch Vakuumdestillation reinigen ließen, wurde auf ihre Isolierung verzichtet. Zur Charakterisierung wurden sie unmittelbar mit Thiamin bei $p_{\mathrm{H}} 8$ umgesetzt und in Alkylthiamindisulfide der Formel

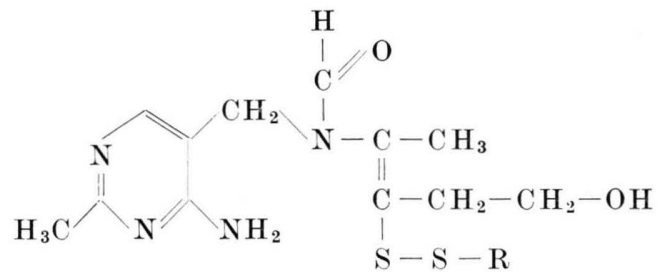

übergeführt (Matsukawa u. a. ${ }^{38}$ ), die sich nun bequem kristallin erhalten ließen. Einige Daten dieser Thiaminderivate sind in Tab. 1 zu finden.

Abb. 4 zeigt den zeitlichen Verlauf der enzymatischen Spaltung der Alkyl-L-cysteinsulfoxyde bei $p_{\mathrm{H}} 8,5$.

Wie aus dem Verlauf der Kurven zu sehen ist, erfolgt bei $(+) S$-Allyl-L-cysteinsulfoxyd (Alliin) eine rasche und nahezu vollständige Spaltung, wäh-

38 T. Matsukawa, H. Kawasaki, T. Iwatsu u. S. Yurugi, J. Vitaminology 1, 13 [1954]. rend bei den synthetischen Sulfoxyd-Derivaten, die am $S$-Atom racemisch sind, die Spaltung bis $50 \%$ rasch erfolgt, von hier an jedoch sehr langsam weiterschreitet. Ähnliches hatten Stoll und SeEBeck auch bei der Alliinase beobachtet. Beide Enzyme scheinen demnach die dem natürlichen Alliin oder (+)S-Methyl-L-cysteinsulfoxyd aus Brassica-Arten („Brassicin“) entsprechende Sulfoxyd-Form zu bevorzugen.

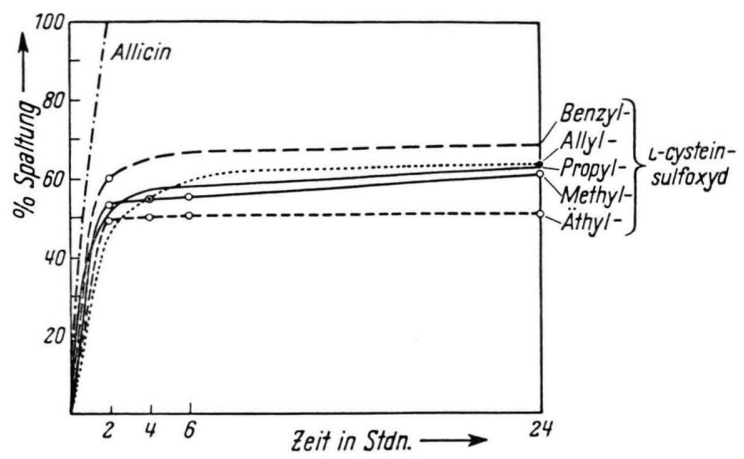

Abb. 4. Zeitlicher Verlauf der enzymatischen Spaltung von $S$-Alkyl-L-cysteinsulfoxyden. $n / 20$-Lösungen in Boratpuffer $p_{\mathrm{H}} 8,5 ; 0,5 \%$ Rohenzym; Raumtemperatur.

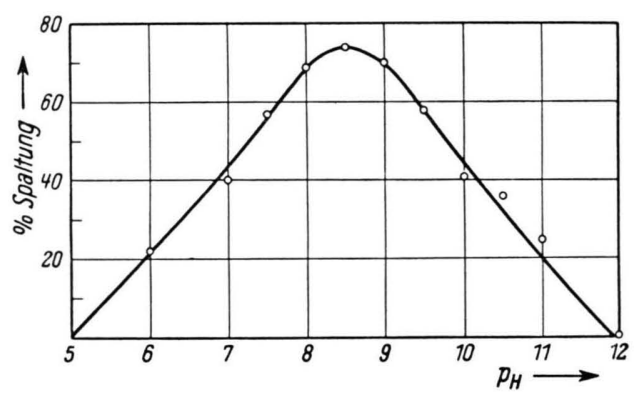

Abb. 5. Abhängigkeit der Aktivität des Albizzia-Enzyms vom $p_{\mathrm{H}}$-Wert. Einzelansätze mit je $200 \mathrm{mg} S$-Methyl-L-cystein und $200 \mathrm{mg}$ Rohenzym in $20 \mathrm{ml}$ Boratpuffer $p_{\mathrm{H}} 8,5$; Raumtemperatur. Versuchsdauer 4 Stunden.

Lanthioninsulfoxyd wurde - vielleicht auf Grund seiner geringen Löslichkeit in Wasser - nicht angegriffen.

Mit der hier aufgeführten, begrenzten Anzahl von Derivaten des L-Cysteins ist sicher die Zahl der spaltbaren Verbindungen nicht erschöpft. Es ist anzunehmen, daß auch die $S$-Alkyl-L-cysteine und $S$ Alkyl-L-cysteinsulfoxyde mit höheren Homologen der Paraffinreihe gespalten werden.

Interessant wäre noch eine Prüfung von Cysteinderivaten, bei denen am $S$-Atom aromatische oder heterocyclische Substituenten sowie Alkenreste sit. zen. 


\begin{tabular}{|c|c|c|c|}
\hline & & 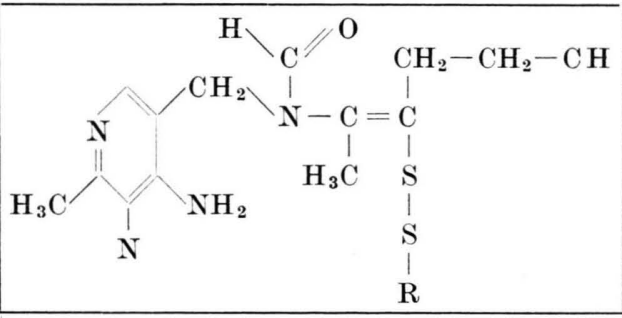 & \\
\hline Ausgangsaminosäure & $\begin{array}{l}\text { Enzymatisches } \\
\text { Spaltprodukt }\end{array}$ & $\begin{array}{l}\text { Reaktionsprodukt } \\
\text { mit Thiamin; } R=\end{array}$ & $\begin{array}{c}\text { Schmp. } \\
\text { (Zers.) }\left[{ }^{\circ} \mathrm{C}\right]\end{array}$ \\
\hline $\begin{array}{l}S \text {-Methyl-L- } \\
\text { cysteinsulfoxyd }\end{array}$ & $\begin{array}{c}\mathrm{CH}_{3}-\mathrm{S}-\mathrm{S}-\mathrm{CH}_{3} \\
\downarrow \\
\mathrm{O}\end{array}$ & $\mathrm{CH}_{3}-$ & 136 \\
\hline $\begin{array}{l}\text { S-Äthyl-L- } \\
\text { cysteinsulfoxyd }\end{array}$ & $\begin{array}{c}\mathrm{C}_{2} \mathrm{H}_{5}-\mathrm{S}-\mathrm{S}-\mathrm{C}_{2} \mathrm{H}_{5} \\
\downarrow \\
\stackrel{\mathrm{O}}{ }\end{array}$ & $\mathrm{C}_{2} \mathrm{H}_{5}-$ & 126 \\
\hline $\begin{array}{l}\text { S-Propyl-L- } \\
\text { cysteinsulfoxyd }\end{array}$ & $\begin{array}{c}\mathrm{C}_{3} \mathrm{H}_{7}-\mathrm{S}-\mathrm{S}-\mathrm{C}_{3} \mathrm{H}_{7} \\
\downarrow \\
\stackrel{\mathrm{O}}{ }\end{array}$ & $\mathrm{C}_{3} \mathrm{H}_{7}-$ & 128 \\
\hline $\begin{array}{l}S \text {-Allyl-L- } \\
\text { cysteinsulfoxyd }\end{array}$ & $\begin{array}{l}\mathrm{CH}_{2}=\mathrm{CH}-\mathrm{CH}_{2}-\mathrm{S} \rightarrow \mathrm{O} \\
\mathrm{CH}_{2}=\mathrm{CH}-\mathrm{CH}_{2}-\mathrm{S}\end{array}$ & $\mathrm{CH}_{2}=\mathrm{CH}-\mathrm{CH}_{2}-$ & 132 \\
\hline $\begin{array}{l}S \text {-Benzyl-L- } \\
\text { cysteinsulfoxyd }\end{array}$ & $-\mathrm{CH}_{2}-\mathrm{S} \rightarrow$ & & 152 \\
\hline
\end{tabular}

Tab. 1. Zusammenstellung der untersuchten $S$-Alkyl-L-cystein-sulfoxyde sowie ihrer enzymatischen Spaltprodukte und der daraus hergestellten Thiaminderivate.

Serin, Homoserin, Homocystein, Methionin und Methyl-sulfonium-methionin werden durch das Albizzia-Enzym nicht angegriffen.

Unsere Untersuchungen über Eigenschaften des Albizzia-Enzyms mußten sich aus technischen Gründen vorläufig auf das Rohenzym beschränken.

Die Untersuchungen brachten folgende Ergebnisse:

a) Das Enzym hat ein $p_{\mathrm{H}}$-Optimum von 8,5 und eine Wirkungsbreite zwischen $p_{\mathrm{H}} 5,5$ und 11,5.

b) Das Enzym hat ein Temperatur-Optimum bei $37^{\circ} \mathrm{C}$. Es wird durch Hitze inaktiviert. Unterhalb $0^{\circ} \mathrm{C}$ findet keine Spaltung des Substrats statt.

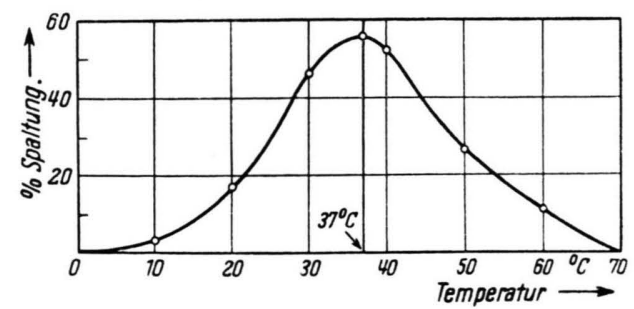

Abb. 6. Abhängigkeit der Aktivität des Albizzia-Enzyms von der Temperatur. Einzelansätze mit je $100 \mathrm{mg} S$-Methyl-Lcystein in $20 \mathrm{ml}$ Boratpuffer $\left(p_{\mathrm{H}} 8,5\right)+50 \mathrm{mg}$ Rohenzym. Versuchsdauer 2 Stunden. c) Das Rohenzym läßt sich ohne Wirkungsverlust (Gegensatz zu Alliinase) aus der wäßrigen Lösung durch Alkohole oder Aceton ausfällen. Im getrockneten Zustand ist es sehr stabil, aber auch Lösungen in Boratpuffer $p_{\mathrm{H}} 8$ lassen sich bei nur mäßigem Verlust an Wirkung monatelang im Eisschrank aufbewahren.

d) Carbonylreagentien hemmen das Enzym. Durch HCN, Hydroxylamin, Hydrazin und Semicarbazid

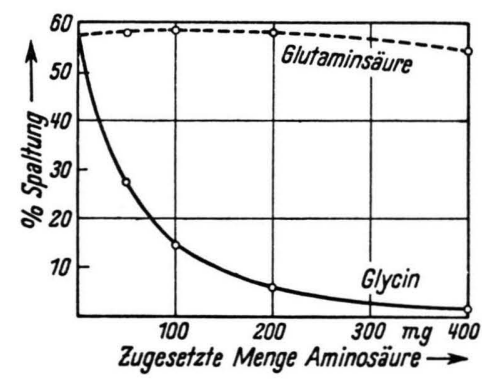

Abb. 7. Einfluß von Glycin und Glutaminsäure auf die Aktivität des Albizzia-Enzyms. Einzelansätze mit je $100 \mathrm{mg}$ $S$-Methyl-L-cystein und $100 \mathrm{mg}$ Rohenzym in $20 \mathrm{ml}$ Boratpuffer $p_{\mathrm{H}} 8,5$; Versuchsdauer 2 Stunden. 
erfolgt vollständige, durch Phenylhydrazin teilweise Hemmung.

e) Zusatz von Glycin zur Reaktionsmischung verringert die Spaltungsrate erheblich. Glutaminsäure dagegen ist ohne nennenswerten Einfluß.

f) Die Aktivität des Albizzia-Rohenzyms läßt sich durch Zusatz von Pyridoxalphosphat steigern.

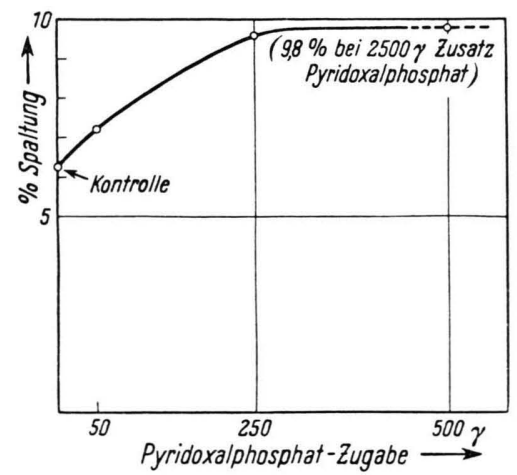

Abb. 8. Einfluß von Pyridoxalphosphat auf die Aktivität des Albizzia-Enzyms. Einzelansätze mit je $200 \mathrm{mg} S$-Methyl-Lcystein in $10 \mathrm{ml}$ Boratpuffer $p_{\mathrm{H}} 8,5 ; 10 \mathrm{mg}$ Rohenzym pro Einzelansatz. Versuchsdauer 2 Stunden. Raumtemperatur.

Abb. 9 zeigt die enzymatische Spaltung von $S$ Methyl-L-cystein in Abhängigkeit von der zugesetzten Menge an Albizzia-Rohenzym.

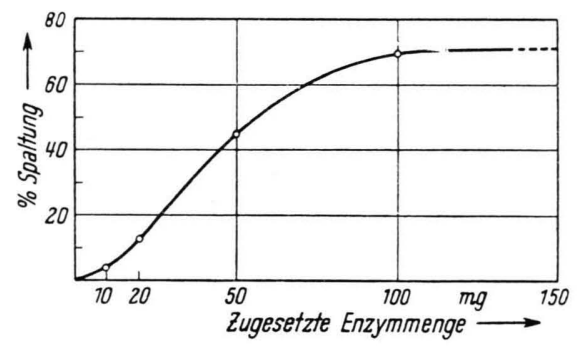

Abb. 9. Einfluß der Enzymmenge auf die Spaltung von $S$-Methyl-L-cystein. Einzelansätze mit je $100 \mathrm{mg} S$-Methyl-Lcystein in $10 \mathrm{ml}$ Boratpuffer $p_{\mathrm{H}} 8,5$. Dauer des Versuchs 2 Stunden.

Aus diesen Resultaten ist zu schließen, daß - ana$\log \mathrm{zu}$ Alliinase und anderen C-S-Lyasen - Py. ridoxalphosphat das Coferment des Albizzia-Enzyms ist.

39 O. Hoffmano-Ostenhof, Enzymologie, Springer-Verlag, Wien 1954.

* Wir danken Herrn Prof. Dr. Markgraf (Botanischer Garten München-Nymphenburg), Herrn T. R. N. Lothiax (Botanischer Garten North Terrace, Adelaide) und dem Jardin E Museu Agricola Do Ultramar (Lissabon) für die freundliche Beschaffung des Samenmaterials.
Systematisch ist das Albizzia-Enzym zu den C-S. Lyasen (Hoffmann-Ostenhof ${ }^{39}$ ) zu zählen. Da es, je nach Substrat, gleichzeitig als Desulfhydrase bzw. Cysteinase, als Thioätherase oder Thionase und als Alkylsulfoxydase fungiert, fällt es schwer, für das Enzym eine geeignete Sammelbezeichnung zu finden. Der Mannigfaltigkeit seiner Substrate wäre am ehesten die Bezeichnung "HS-RS-RSO-Cysteinase“ gerecht.

Die Frage der Nomenklatur ist jedoch erst akut, wenn nach Reindarstellung des Albizzia-Enzyms durch die Methoden der Proteinchemie seine Einheitlichkeit bewiesen worden ist und das einheitliche Enzym dann immer noch die genannten 3 Typen von Spaltungen durchführt.

\section{Beschreibung der Versuche}

\section{Ia. A u sgang smaterial}

Albizzia lophantha-Samen * wurden zunächst grob gemahlen und von den Samenschalen (etwa 50\% des Gesamtgewichts) abgetrennt. Die Endospermteile wurden feinst gemahlen und trocken aufbewahrt.

\section{Ib. Substrate und chemische} Substanzen ${ }^{* *}$

L-Lanthionin und L-Lanthioninsulfoxyd wurden uns durch Prof. H. ZAHN, DL- und allo-Cystathionin, DLHomoserin und Pyridoxalphosphat durch Dr. Y. MAtsuo zur Verfügung gestellt ${ }^{* *}$.

Ic. Die Darstellung von $S$-Methyl-, $S$-Äthyl-, $S$-Propyl-, $S$-Allyl- und $S$-Benzyl---cystein sowie von $S$-MethylDL-cystein erfolgte nach bekannten Methoden.

Durch Oxydation der $S$-Alkyl-cysteine mit 25-proz. $\mathrm{H}_{2} \mathrm{O}_{2}$ in Wasser nach $\mathrm{S}$ Toll und SEEBECK ${ }^{40}$ wurden die entsprechenden, am $S$-Atom racemischen $S$-Alkyl-Lcysteinsulfoxyde dargestellt.

Tab. 2 enthält die Darstellungsmethoden und einige Daten der untersuchten Substanzen.

\section{Id. Darstellung von Alliin}

$[(+) S$-Allyl-L-cysteinsulfoxyd] aus Allium sativum $100 \mathrm{~g}$ enthäutete Zwiebeln von Allium sativum wurden im Starmix mit $300 \mathrm{ml}$ Methanol homogenisiert und die Mischung abgepreßt. Der Preßrückstand wurde nochmals in der gleichen Weise mit 70-proz. Methanol

** Herrn Prof. Dr. H. ZaHN (Deutsches Wollforschungsinstitut, Aachen) und Herrn Dr. Y. Matsuo (School of Medicine, Berkeley, Calif.) danken wir für die großzügige Überlassung wertvoller Substanzen.

40 A. Stoll u. E. Seebeck, Helv. chim. Acta 34, 481 [1951]. 


\begin{tabular}{|c|c|c|c|c|c|c|}
\hline \multirow{2}{*}{ Aminosäure } & \multirow{2}{*}{ Darstellung } & \multirow{2}{*}{ Fp. } & \multirow{2}{*}{ Optische Drehung } & \multicolumn{3}{|c|}{$\begin{array}{l}\text { Rf-Werte, (absteigend, } \\
\text { Schl. \& Sch. 2043 b) }\end{array}$} \\
\hline & & & & BEW 4:1:1 & $\underset{35}{\text { Pyridin }}$ & $\begin{array}{l}- \text { Butanol } \\
: \quad 35\end{array}$ \\
\hline Djenkolsäure & $\mathrm{a}$ & & & 0,06 & & 0,05 \\
\hline $\begin{array}{l}S \text {-Methyl-L- } \\
\text { cystein }\end{array}$ & b c & $\begin{array}{l}216^{\circ} \mathrm{C} \\
\text { (Zers.) }\end{array}$ & $\begin{array}{l}{[\alpha]_{\mathrm{D}}^{23}-23,6} \\
\left(c=1 ; \mathrm{H}_{2} \mathrm{O}\right)\end{array}$ & 0,35 & & 0,35 \\
\hline $\begin{array}{l}\text { S-Methyl-DL- } \\
\text { cystein }\end{array}$ & $\mathrm{b}$ & $\begin{array}{l}216^{\circ} \mathrm{C} \\
\text { (Zers.) }\end{array}$ & - & 0,35 & & 0,35 \\
\hline $\begin{array}{l}S \text {-Äthyl-L- } \\
\text { cystein }\end{array}$ & $\mathrm{b} \mathrm{d}$ & $\begin{array}{l}260^{\circ} \mathrm{C} \\
\text { (Zers.) }\end{array}$ & $\begin{array}{l}{[\alpha]_{\mathrm{D}}^{23}-22,8} \\
\left(c=5 ; \mathrm{H}_{2} \mathrm{O}\right)\end{array}$ & 0,49 & & 0,45 \\
\hline $\begin{array}{l}\text { S-Propyl-L- } \\
\text { cystein }\end{array}$ & $\mathrm{e}$ & $\begin{array}{l}242-243^{\circ} \mathrm{C} \\
\text { (Zers.) }\end{array}$ & $\begin{array}{c}{[\alpha]_{\mathrm{D}}^{23}-24,9} \\
\left(c=2,5 ; \mathrm{H}_{2} \mathrm{O}\right)\end{array}$ & 0,60 & & 0,55 \\
\hline $\begin{array}{l}S \text {-Allyl-L- } \\
\text { cystein }\end{array}$ & $\mathrm{e}$ & $\begin{array}{l}218^{\circ} \mathrm{C} \\
\text { (Zers.) }\end{array}$ & $\begin{array}{c}{[\alpha]_{\mathrm{D}}^{23}-16,4} \\
\left(c=2,5 ; \mathrm{H}_{2} \mathrm{O}\right)\end{array}$ & 0,56 & & 0,51 \\
\hline $\begin{array}{l}\text { S-Benzyl-L- } \\
\text { cystein }\end{array}$ & $\mathrm{f}$ & $\begin{array}{c}215-216^{\circ} \mathrm{C} \\
\text { (Zers.) }\end{array}$ & $\begin{array}{c}{[\alpha]_{\mathrm{D}}^{23}+19} \\
(c=5 ; 1 n \cdot \mathrm{NaOH})\end{array}$ & 0,72 & & 0,69 \\
\hline $\begin{array}{l}\text { S-Methyl-L- } \\
\text { cysteinsulfoxyd }\end{array}$ & $\mathrm{e}$ & $\begin{array}{l}163^{\circ} \mathrm{C} \\
\text { (Zers.) }\end{array}$ & $\begin{array}{l}{[\alpha]_{D}^{23}-28,8} \\
\left(c=5 ; \mathrm{H}_{2} \mathrm{O}\right)\end{array}$ & 0,15 & & 0,18 \\
\hline $\begin{array}{l}S \text {-Äthyl-L- } \\
\text { cysteinsulfoxyd }\end{array}$ & $\mathrm{e}$ & $\begin{array}{l}161^{\circ} \mathrm{C} \\
\text { (Zers.) }\end{array}$ & $\begin{array}{l}{[\alpha]_{\mathrm{D}}^{23}-16,6} \\
\left(c=5 ; \mathrm{H}_{2} \mathrm{O}\right)\end{array}$ & 0,24 & & 0,24 \\
\hline $\begin{array}{l}S \text {-Propyl-L- } \\
\text { cysteinsulfoxyd }\end{array}$ & $\mathrm{e}$ & $\begin{array}{l}150-152^{\circ} \mathrm{C} \\
\text { (Zers.) }\end{array}$ & $\begin{array}{l}{[\alpha]_{\mathrm{D}}^{23}-11} \\
\left(c=2 ; \mathrm{H}_{2} \mathrm{O}\right)\end{array}$ & 0,32 & & 0,31 \\
\hline $\begin{array}{l}S \text {-Allyl-L- } \\
\text { cysteinsulfoxyd }\end{array}$ & $\mathrm{e}$ & $\begin{array}{c}146-147^{\circ} \mathrm{C} \\
\text { (Zers.) }\end{array}$ & $\begin{array}{c}{[\alpha]_{\mathrm{D}}^{23}-10} \\
\left(c=0,8 ; \mathrm{H}_{2} \mathrm{O}\right)\end{array}$ & 0,28 & & 0,29 \\
\hline $\begin{array}{l}S \text {-Benzyl-L- } \\
\text { cysteinsulfoxyd }\end{array}$ & $\mathrm{e}$ & $\begin{array}{c}158-159^{\circ} \mathrm{C} \\
\text { (Zers.) }\end{array}$ & $\begin{array}{c}{[\alpha]_{\mathrm{D}}^{23} 0} \\
(c=2,1 ; 1 n \cdot \mathrm{NaOH})\end{array}$ & 0,49 & & 0,47 \\
\hline
\end{tabular}

a loc. cit. ${ }^{3}$

b V. du Vigneaud, H. S. Loring u. H. A. Craft, J. biol. Chemistry 105, 481

[1934].
c H. T. Clarke u. J. M. Inouye, J. biol. Chemistry 94, 541 [1931/32]. d M. D. Armstrong u. J. D. Lewis, J. org. Chemistry 16, 749 [1951].

e loc. cit. ${ }^{5 a}$

f V. Du Vigneaud, L. F. Audrieth u. H. S. Loring, J. Amer. chem. Soc. 52, 4500 [1930].

Tab. 2. Darstellungsmethoden und einige Daten der verwendeten Substrate.

behandelt. Die Filtrate wurden i. V. eingedampft, in $500 \mathrm{ml}$ Wasser aufgenommen und die Lösung filtriert. Das Filtrat wurde durch eine Säule mit $50 \mathrm{ml}$ Lewatit S 100 (H-Form) geschickt. Nach Waschen der Säule mit dest. Wasser wurde mit 2-proz. Ammoniak eluiert. Die alliinhaltigen Fraktionen wurden i. V. eingeengt. Nach Auflösen des •Rückstandes in heißem 70-proz. Methanol, dem Aceton bis zur Trübung zugesetzt wurde, schied sich Alliin in feinen, langen Nadeln aus. Umkristallisation aus verdünntem Aceton. Ausbeute $108 \mathrm{mg}$ Alliin [Schmp. $163-165^{\circ} \mathrm{C}$ (Zers.)]. Papierchromatographische Prüfung zeigte, daß das Präparat Spuren von $S$-Methyl-L-cysteinsulfoxyd enthielt.

II. Isolierung von D jenkolsäure a u s den Samen von Albizzialophantha Benth.

a) $100 \mathrm{~g}$ feinst gemahlene Samen von Albizzia lophanta wurden 3-mal mit je $500 \mathrm{ml} \mathrm{50-proz.} \mathrm{Methanol}$ ausgekocht. Die Filtrate wurden von Methanol befreit, mit Wasser auf $1 l$ aufgefüllt und filtriert. Das Filtrat wurde auf eine Säule mit $100 \mathrm{ml}$ Lewatit S 100 (HForm) gebracht. Nach Waschen der Säule mit dest. Wasser wurde mit 2-proz. Ammoniak eluiert. Das gesamte Eluat wurde eingedampft. Bei vorsichtiger Wasserzugabe blieb ein weißer Niederschlag zurück, der abfiltriert und getrocknet wurde. Ausbeute $150 \mathrm{mg}$.

b) Wegen der geringen Ausbeute nach Methode II a wurden weitere $100 \mathrm{~g}$ Samen nach vaN VEeN und Hyman $^{41}$ aufgearbeitet.

Ausbeute 1,6 g Djenkolsäure.

$\mathrm{C}_{7} \mathrm{H}_{14} \mathrm{~N}_{2} \mathrm{~S}_{2} \mathrm{O}_{4}$ Ber. C 33,06 H 5,54 N 11,01 S 25,21. Gef. C 32,43 H 8,76 N 11,61 S 23,04.

41 A. G. van Veen u. A. J. Hyman, loc. cit. ${ }^{1 b}$. 
III. Papier chromatographis cher $\mathrm{Nachweis}$ der D jenkolsäure-Spaltung durch ein Enzym der Albizzia-Samen

Ansatz a. $1 \mathrm{~g}$ fein gepulverte Samen wurde mit $10 \mathrm{ml}$ Wasser kalt angerührt.

Ansatz $b .1 \mathrm{~g}$ fein gepulverte Samen wurde mit $10 \mathrm{ml}$ 50-proz. Methanol aufgekocht.

Ansatzc. $10 \mathrm{mg}$ synthetische Djenkolsäure wurden in $5 \mathrm{ml}$ Boratpuffer $p_{\mathrm{H}} 8,5$ aufgeschlämmt und mit $100 \mathrm{mg}$ Rohenzym versetzt.

Von den Ansätzen a, b und c wurden nach 0 Stdn. und nach 6 Stdn. je $20 \mu \mathrm{l}$ auf Bogen von Schleicher \& Schüll $2043 \mathrm{~b}$ aufgetragen.

Entwicklungsflüssigkeit: Butanol-Essigsäure-Wasser $4: 1: 1$. Absteigende Chromatographie. Sichtbarmachung durch Besprühen der getrockneten Chromatogramme mit Ninhydrinlösung (vgl. Abb. 1).

In gleicher Weise wie bei III c wurde mit Cystathionin verfahren (vgl. Abb. 2).

\section{Darstellung des Albizzia- Rohenzyms}

a) $100 \mathrm{~g}$ feinst gepulverte Samen von Albizzia lophantha wurden mit $400 \mathrm{ml}$ Wasser angerührt. Nach mehrstdg. Stehen unter wiederholtem Umschütteln wurde die Mischung scharf zentrifugiert. Der abdekantierte, milchige Überstand wurde mit der 5 -fachen Menge 96-proz. Alkohols versetzt und die Mischung zentrifugiert. Der schwach gelbliche Bodensatz wurde nach Abgießen der überstehenden Flüssigkeit 3-mal mit Aceton verrieben, abgesaugt und auf Tonteller getrocknet. Ausbeute $15 \mathrm{~g}$ Rohenzym: weißes, lockeres, nicht hygroskopisches Pulver.

b) Mit dem Rückstand des Samenpulvers wurde die Operation wiederholt. Ausbeute: $4 \mathrm{~g}$ eines gelblichgrauen Rohenzyms von geringerer Aktivität als unter a.

$$
\begin{gathered}
\text { V. Durchführug der Spaltungs- } \\
\text { reaktionen und Nachweis der } \\
\text { enzymatis gebildeten } \\
\text { Spaltprodukte }
\end{gathered}
$$

$100-200 \mathrm{mg}$ der Aminosäuren wurden in $10-20 \mathrm{ml}$ Boratpuffer $p_{\mathrm{H}} 8,5$ gelöst und die $p_{\mathrm{H}}$-Werte durch $\mathrm{Zu}$ gabe von NaOH-Lösung auf $p_{\mathrm{H}} 8,5$ eingestellt. Zugabe von $100 \mathrm{mg}$ Albizzia-Rohenzym und Umschütteln bis zur Lösung des Enzyms.

\section{a) Brenztraubensäure-Nachweis (qualitativ)}

Die Reaktionsmischung wurde mit dem gleichen Volumen einer 20-proz. Trichloressigsäure-Lösung versetzt und filtriert. Zugabe zum Filtrat einer heißen 2-proz. Lösung von Dinitrophenylhydrazin in verdünnter Salzsäure. Die entstandene Fällung wurde nach 2 -stdg. Stehen im Eisschrank abfiltriert und aus Eisessig umkristallisiert.

Brenztraubensäure-Dinitrophenylhydrazon: Schmp. $215-216^{\circ} \mathrm{C}$.

b) Brenztraubensäure-Bestimmung (quantitativ)

Die Bestimmung erfolgte nach Stoll und Seebeck ${ }^{42}$.

42 A. Stoll u. E. Seebeck, l. c. 5 b.

\section{c) Ammoniak-Nachweis (qualitativ)}

Die Reaktionsmischung wurde mit einem Tropfen Silikonöl versetzt und destilliert. Im alkalisch reagierenden Destillat wurde $\mathrm{NH}_{3}$ durch $\mathrm{N}$ e $\beta$ le r s Reagenz nachgewiesen.

d) Ammoniak-Nachweis (quantitativ)

Ein nach c erhaltenes Destillat wurde in einer gemessenen Menge $n / 10$-HCl-Lösung aufgefangen. Rücktitration mit $n / 10-\mathrm{NaOH}$-Lösung gegen Methylrot.

\section{e) Nachweis gebildeter Thiole}

1. Die Reaktionsmischung wurde mit einigen Tropfen einer Jodazid-Lösung (Lösung von $100 \mathrm{mg}$ Natriumazid in einer Mischung aus gleichen Teilen Alkohol und $n / 10$-Jodlösung) versetzt. Die Reaktion wurde als positiv angesehen, wenn unter stürmischer Gasentwicklung spontane Entfärbung eintrat.

2. Zugabe einer alkalischen Lösung von Nitroprussid-Natrium: rotviolette Färbung bei Anwesenheit freier SH-Gruppen.

3. Geruchsprobe:

$\mathrm{CH}_{3}-\mathrm{SH}$ und $\mathrm{C}_{2} \mathrm{H}_{5}-\mathrm{SH}$

$\mathrm{C}_{3} \mathrm{H}_{7}-\mathrm{SH}$ und Allyl-SH

„kohlartig“,

„knoblauchartig“

\section{4. $\mathrm{H}_{2} \mathrm{~S}$-Nachweis}

Destillation der Reaktionsmischung bei schwach saurem $p_{\mathrm{H}}$ und Auffangen des Destillats in $\mathrm{AgNO}_{3}$ - oder $\mathrm{Pb}\left(\mathrm{NO}_{3}\right)_{2}$-Lösung.

5. Nachweis von Merkaptanen

Destillation der Reaktionsmischung bei schwach saurem $p_{\mathrm{H}}$ und Auffangen des Destillats in $\mathrm{HgCl}_{2}$ - oder Kupferacetat-Lösung.

\section{Isolierung von}

Benzylthiosulfinsäurebenzylester

$1 \mathrm{~g} S$-Benzyl-L-cysteinsulfoxyd wurde in $100 \mathrm{ml} \mathrm{Bo-}$ ratpuffer $p_{\mathrm{H}} 8,5$ aufgelöst. Zugabe von $1 \mathrm{~g}$ Rohenzym. Nach wenigen Min. setzte Ausscheidung von Benzylthiosulfinsäurebenzylester ein, die nach etwa 4 Stdn. beendet war. Der Niederschlag wurde abfiltriert, mit Wasser gewaschen und nach Trocknen aus Äther-Petroläther umkristallisiert.

$0,46 \mathrm{~g}$ schöne, durchsichtige rhombische Kristalle von brennend-scharfem Geschmack. Schmp. $81-83^{\circ} \mathrm{C}$. $\begin{array}{lllll}\mathrm{C}_{14} \mathrm{H}_{14} \mathrm{OS}_{2} & \text { Ber. C 64,08 } & \text { H 5,39 } & \text { S 24,44. }\end{array}$ Gef. C 63,85 H 5,39 S 24,00.

VII. Umsetzung von

Alkylthiosulfinsäurealkylestern mit Thiamin

1 g S-Methyl-(Äthyl-, Propyl-, Allyl- und Benzyl-) Lcysteinsulfoxyd wurde in Boratpuffer $p_{\mathrm{H}} 8,5$ aufgelöst. Zugabe von $0,5 \mathrm{~g}$ des Rohenzyms. Die Mischung wurde unter wiederholtem Umschütteln $4 \mathrm{Stdn}$. bei Raumtemperatur gehalten. 3-maliges Ausschütteln mit Chloroform und Trocknen der Chloroformphasen über $\mathrm{Na}_{2} \mathrm{SO}_{4}$. Nach Abdestillieren des Chloroforms i. V. blieben farblose Öle zurück, die brennend scharf schmeckten. Sie wurden in wenig Alkohol gelöst und zu einer neutralisierten Lösung von Thiamindihydrochlo- 
rid gegeben. Die Umsetzung erfolgte nach $\mathrm{M}_{\text {Atsukawa }}{ }^{43}$ u. a. (Schmp. s. Tab. 1).

VIII. Bestimmung der Enzymaktivität gegenüber verschiedenen Substraten

Von $S$-Methyl-(Äthyl-, Propyl-, Allyl- und Benzyl-) Lcystein bzw. den entsprechenden Sulfoxyden wurden $25 \mathrm{ml}$ einer 1/20-molaren Lösung in Boratpuffer $p_{\mathrm{H}} \mathbf{8 , 5}$ mit je $100 \mathrm{mg}$ Rohenzym versetzt und bei Raumtemperatur stehen gelassen. Nach 2, 4, 6 und 24 Stdn. wurden mit je $5 \mathrm{ml}$ der einzelnen Ansätze Brenztraubensäure gravimetrisch oder Ammoniak titrimetrisch bestimmt.

IX. Bestimmung der Enzymaktivität unter verschiedenen Bedingungen (Pyridoxylphosphat-Zusatz, unter. schiedliche Temperaturen, Glycin und Glutaminsäure-Zusatz, verschiedene $p_{\text {H }}-$ Werte, Inhibitoren)

Die einzelnen Bestimmungen wurden wie unter VIII. durchgeführt. Abänderungen in den Einwaagen und Versuchsbedingungen sind aus den jeweiligen Abbildungen zu entnehmen.

43 T. Matsukawa, l. c. ${ }^{38}$.
X. Gewinnung von $S$ - Methyl-D-cystein a u s S-Methyl-DL-cystein

$2 \mathrm{~g} S$-Methyl-DL-cystein wurden in $100 \mathrm{ml}$ Boratpuffer $p_{\mathrm{H}} 8,5$ gelöst und mit $1 \mathrm{~g}$ Rohenzym versetzt. Die Mischung wurde $6 \mathrm{Stdn}$. bei $35^{\circ} \mathrm{C}$ gehalten, abschlieBend kurz zum Sieden erhitzt und von ausgeschiedenem Enzym abfiltriert. Nach Auffüllen der Mischung mit Wasser auf etwa $500 \mathrm{ml}$ wurde die Lösung durch eine Säule mit $50 \mathrm{ml}$ Zeo Carb 215 (H-Form) geschickt. Der Gesamt-Durchlauf wurde zur Bestimmung gebildeter Brenztraubensäure mit überschüssiger Dinitrophenylhydrazin-Lösung in verdünnter Salzsäure versetzt. Die Bestimmung ergab eine 40-proz. Spaltung bezogen auf die DL-Form.

Die Säule wurde anschließend mit Wasser nachgewaschen und dann mit 2-proz. Ammoniak eluiert. Das Eluat wurde i. V. eingedampft und der Rückstand aus verdünntem Alkohol umkristallisiert. Glitzernde Kristallblättchen. Schmp. $220^{\circ} \mathrm{C}$ (Zers.). Ausbeute $0,35 \mathrm{~g}$ (35\% der Theorie).

$\mathrm{C}_{4} \mathrm{H}_{9} \mathrm{O}_{2} \mathrm{NS} \quad$ Ber. C $35,6 \quad \mathrm{H} 6,67 \quad \mathrm{~N} \mathrm{10,37} \quad \mathrm{S} \mathrm{23,7.}$

Gef. C 35,62 H 6,64 N 10,0 S 22,15.

Optische Drehung: $[\alpha]{ }_{\mathrm{D}}^{26}-28,1^{\circ}\left(c=2 ; \mathrm{H}_{2} \mathrm{O}\right)$

Die Mikroanalysen wurden im mikroanalytischen Laboratorium A. BERnhardt, Max-Planck-Institut für Kohlenforschung, Mülheim/Ruhr durchgeführt.

\title{
Untersuchungen am Vaccine-Virus
}

V. Mitt.: Elektronenoptische Studie über den Pepsin-Abbau des Elementarkörpes bei Variation von Fixierung und $p_{\mathrm{H}}$-Wert

\section{Von Dietrich Peters}

Aus der Abteilung für Virusforschung des Bernhard Nocht-Institutes für Schiffs- und Tropenkrankheiten, Hamburg (Direktor: Prof. Dr. Dr. h. c. E. G. Nauck)

(Z. Naturforschg. 12 b, 697-703 [1957] ; eingegangen am 3. Juli 1957)

\begin{abstract}
Frühere Angaben über den Aufbau des Elementarkörpers ließen sich durch Anwendung weiterer Fixierungsmethoden stützen. Die durch Osmiumtetroxyd induzierte Resistenz der Elementarkörper gegenüber Pepsin wird durch Zugabe von L-Cystein aufgehoben. Der im Zentrum der DNS-haltigen Ringstruktur liegende Körper ist besonders im schwach sauren Bereich $\left(p_{\mathrm{H}} 3,0-4,5\right)$ gegen Pepsin resistenter als die periphere Schicht; er ist daher unter diesen Bedingungen regelmäßig sichtbar.
\end{abstract}

Vorausgegangene Studien hatten ergeben, daß nach $\mathrm{Chabaud-Fixierung}$ und Pepsinbehandlung die Desoxyribonucleo-Proteide (DN-Proteide) der Elementarkörper (Elk.) des Vaccine- ${ }^{1}$ und des Molluscum contagiosum-Virus ${ }^{2}$ vorwiegend ringförmig angeordnet sind. Sie sind demnach nicht Bestandteil der in unbehandelten Partikeln vorhandenen zentralen Verdichtung (s. Abb. 3); letztere wird durch Pepsin unter den angegebenen Normalbedingungen

1 D. Peters u. W. Stoeckenius, Proc. 3rd. Int. Conf. Electron Microscopy, London 1954, S. 237, 1956; W. Sтовскеnius u. D. Peters, Z. Naturforschg. 10 b, 77 [1955]. weitgehend abgebaut. Trotz der Empfindlichkeit gegenüber Pepsin können Reste dieser Verdichtung neben dem desoxyribonucleinsäure(DNS)-haltigen Material sichtbar bleiben, wenn man den Pepsinabbau nur unvollständig ablaufen läßt. Es findet sich dann deutlich differenziert am Ort der zentralen Aufhellung des DNS-haltigen Ringes ein runder Körper. Der Elk. des Vaccine-Virus besteht demzufolge aus mindestens vier strukturell unterscheid-

2 D. Peters u. W. Stoeckenius, Z. Tropenmed. Parasitol. 5, 329 [1954]. 